\title{
1 Gut microbiota features associated with Clostridioides 2 difficile colonization in dairy calves
}

3

5

6

7

8

9

10

11

12

13

14

15

16

17

18

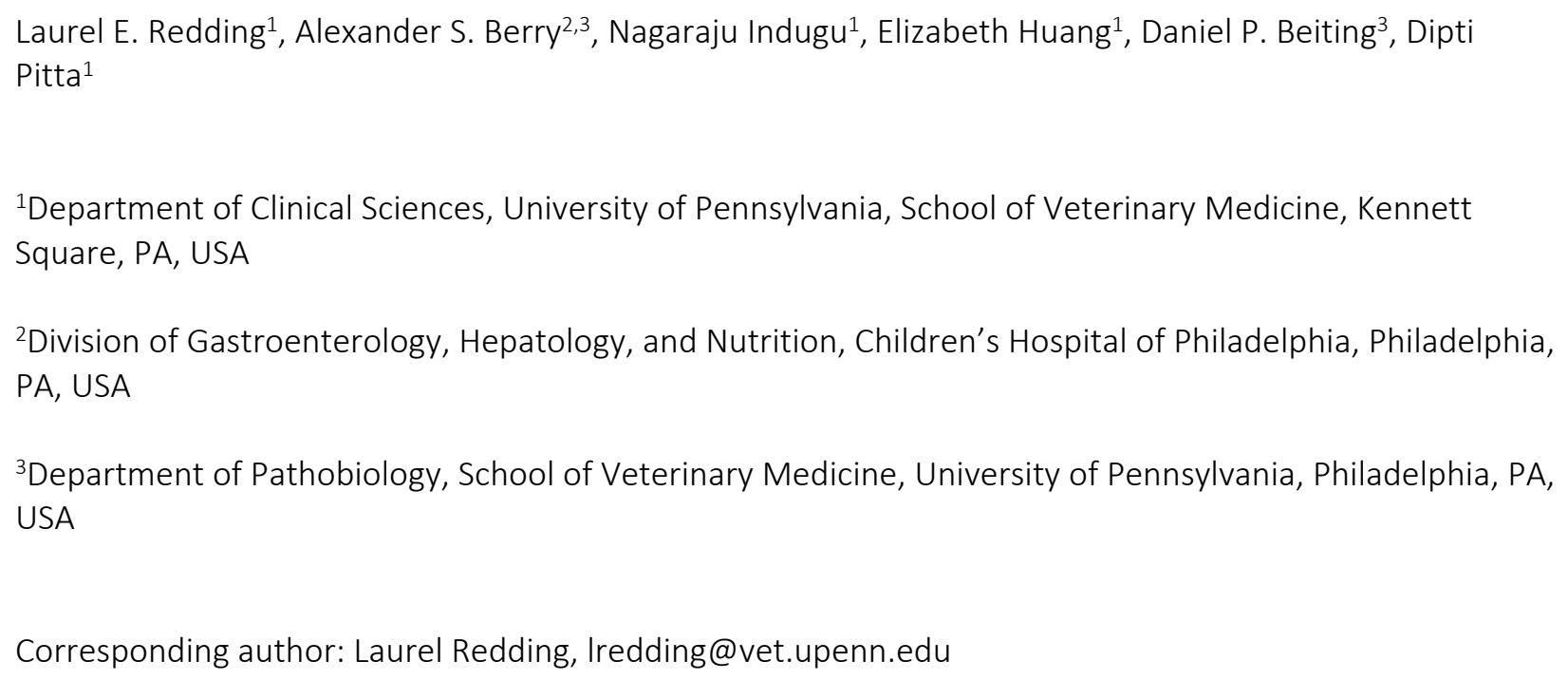




\section{Abstract}

21 Diarrheal disease, a major cause of morbidity and mortality in dairy calves, is strongly associated with the

22 health and composition of the gut microbiome. Clostridioides difficile is an opportunistic pathogen that

23 proliferates and can produce enterotoxins when the host experiences gut dysbiosis. However, even

24 asymptomatic colonization with $C$. difficile can be associated with differing degrees of microbiome

25 disruption in a range of species, including people, swine, and dogs. Little is known about the interaction

26 between $C$. difficile and the gut microbiome in dairy calves. In this study, we sought to define microbial

27 features associated with $C$. difficile colonization in pre-weaned dairy calves less than 2 weeks of age. We

28 characterized the fecal microbiota of 80 calves from 23 different farms using 16 S rRNA sequencing and

29 compared the microbiota of $C$. difficile-positive $(n=24)$ and C. difficile-negative calves $(n=56)$. Farm

30 appeared to be the greatest source of variability in the gut microbiota. When controlling for calf age, diet,

31 and farm location, there was no significant difference in Shannon alpha diversity $(P=0.50)$ or in weighted

32 UniFrac beta diversity $(\mathrm{P}=0.19)$ between $C$. difficile-positive and -negative calves. However, there was a

33 significant difference in beta diversity as assessed using Bray-Curtiss diversity $(P=0.0077)$, and $C$. difficile-

34 positive calves had significantly increased levels of Ruminococcus (gnavus group) (Adj. $P=0.052$ ),

35 Lachnoclostridium (Adj. $P=0.060$ ), Butyricicoccus (Adj. $P=0.060$ ), and Clostridium sensu stricto 2 compared

36 to C. difficile-negative calves. Additionally, C. difficile-positive calves had fewer microbial co-occurrences

37 than C. difficile-negative calves, indicating reduced bacterial synergies. Thus, while C. difficile colonization

38 alone is not associated with dysbiosis and is therefore unlikely to result in an increased likelihood of

39 diarrhea in dairy calves, it may be associated with a more disrupted microbiota. 


\section{Introduction}

Infectious diarrheal disease is one of the main causes of mortality in dairy calves $(1,2)$, and calves

43 less than 30 days of age are at highest risk of developing diarrhea $(3,4)$. Studies have shown that gut

44 microbial composition is associated with gut health and the likelihood of diarrhea: reductions in microbial

45 diversity are associated with an increased incidence of diarrhea (5), and the colonization of the calf gut

46 with beneficial bacteria along with the decreased colonization of potential pathogens decreases the

47 likelihood of calf diarrhea (6).

48 Clostridioides difficile is a spore-forming anaerobic, gram-positive bacillus that is a significant

49 enteric pathogen in many species of animals. Colonization with $C$. difficile has been shown to be

50 associated with reduced gut microbial diversity and increased colonization of pathogenic bacteria in

51 people $(7,8)$, and we recently demonstrated a similar association in puppies (9). Dairy calves, like the

52 neonates of other species, are colonized with $C$. difficile at high rates, with reported prevalences ranging

53 from $28-56 \%(10,11)$. While there is some evidence that infection with $C$. difficile can result in diarrhea in

54 calves (12), the effect of the asymptomatic colonization of calves on the gut microbiome is unknown.

55 Given the crucial role of the gut microbiome in providing colonization resistance against pathogens that

56 cause diarrhea $(13,14)$, a better understanding of the effect of pathogens such as $C$. difficile on the calf

57 gut microbiome is needed. The goal of this study was thus to define the gut microbiota features

58 associated with $C$. difficile colonization in dairy calves and to define the effects of calf age, diet, and farm 59 on the risk of colonization.

60

\section{Methods}

62 Sample collection: Fecal samples were manually collected from up to five randomly selected healthy

63 calves less than two weeks of age from each of 23 dairy farms in Pennsylvania, Maryland and Delaware. 
64 This study was approved by the Institutional Animal Care and Use Committee of the University of

65 Pennsylvania.

66

67 Detection of $C$. difficile:

69 assay; Cepheid, Sunnyvale, CA, USA) according to the manufacturer's instructions. This assay detects the

70 cytotoxin gene $(t c d B)$ and binary toxin genes ( $c d t A$ and $c d t B)$. Additionally, the assay has a callout for

71 ribotype NAP1/B1/027.

72 To rule out the possibility of colonization with non-toxigenic $C$. difficile, pooled fecal samples from

73 each farm were also submitted for anaerobic culture. Briefly, $0.5 \mathrm{~g}$ of formed fecal sample was mixed with

$740.5 \mathrm{ml}$ of $100 \%$ ethanol. The mixture remained for 60 minutes at room temperature before being

75 inoculated on Cycloserine-cefoxitin fructose modified agar (CCFA) (Remel ${ }^{\mathrm{TM}}$ ) or Clostridium difficile

76 Selective Agar (BBL ${ }^{\mathrm{TM}}$ ) and Columbia CNA agar (Thermo Fisher Scientific Remel Products). Inoculated

77 plates and broth were incubated in BD Gas-Pak ${ }^{\mathrm{TM}}$ EZ container systems with BD BBL ${ }^{\mathrm{TM}}$ CO2 generators and

$78 \mathrm{BD} \mathrm{BBL}{ }^{\mathrm{TM}}$ Gas Pak ${ }^{\mathrm{TM}}$ anaerobic $\mathrm{CO} 2$ indicators (Franklin Lakes, NJ) at $36^{\circ} \mathrm{C} \pm 2^{\circ} \mathrm{C}$ under anaerobic growth

79 conditions for seven days and checked for growth every other day. Suspect colonies were identified and

80 isolated. Isolates were confirmed to be $C$. difficile by Maldi-TOF identification and/or RapID ANA II System

81 (Thermo Fisher Scientific Remel Products).

82

$83 \quad 16 S$ rRNA sequencing

84 DNA was extracted from fecal samples using Qiagen PowerSoil DNA extraction kit. 16S rRNA sequencing

85 was performed as described previously $(9,15)$. Briefly, the $V 4$ region of the $16 S$ rRNA gene was amplified

86 using PCR, which was performed using Accuprime Pfx Supermix and custom primers for 30 cycles (15).

87 PicoGreen quantification was used to normalize post-PCR products and AMPureXP beads were used to 
88 clean the combined pools. Libraries were quantified and sized using a Qubit 2.0 and Tapestation 4200,

89 respectively. $250 \mathrm{bp}$ paired-end sequencing was performed using an Illumina MiSeq.

90

91 Sequence data processing using QIIME2

92 The QIIME2 pipeline (16) was used to process and analyze 165 sequencing data. Samples were

93 demultiplexed using q2-demux and denoised using Dada2 (17). Sequences were aligned using maaft (18)

94 and phylogenetic trees were reconstructed using fasttree (19). Shannon alpha diversity, weighted UniFrac

95 and Bray-Curtis beta diversity metrics were estimated using q2-core-metrics-diversity after samples were

96 rarefied to 1941 reads per sample, and p-values were adjusted for multiple hypothesis testing using

97 Benjamini-Hochberg (B-H) false discovery rate (FDR) corrections (20). Taxonomy was assigned to

98 sequences using q2-feature-classifier classify-sklearn (21) against the Silva reference database (22). Taxa

99 were collapsed to the genus level, when possible. OTUs with less than $1 \%$ average relative abundance

100 across all samples were removed.

101

102 Correlation analysis and differential feature selection

The correlation between $C$. difficile culture status and Shannon alpha diversity was determined

104 using a linear mixed effects model as implemented in the Ime4 package (23) in R where age was

105 controlled for as a fixed effect and with farm and diet as random effects. The correlation between $C$.

106 difficile culture status on gut microbiota beta diversity was determined using PERMANOVA as

107 implemented in the vegan package (24) in R controlling for age, farm, and diet. Principal coordinate

108 analyses were performed using the phyloseq package in R (25). Differentially-abundant taxa were

109 determined using LDA Effect Size (LEfSe) (26) and Analysis of Composition of microbiomes (ANCOM), and

$110 \mathrm{p}$-values were adjusted for multiple hypothesis testing using B-H FDR corrections in R. The Dice index (27) 
111 was used to determine the co-occurrence of bacterial genera. Boxplots and LEfSe plots were visualized

112 using ggplot2 (28) and ggthemes.

\section{Results:}

\section{Subject characteristics and C. difficile status}

116 Fecal samples were collected from a total of 92 Holstein calves from 23 farms. All calves appeared

117 systemically healthy at the time of sampling and none had received antimicrobial therapy. The mean (SD)

118 age of the calves was 7.0 (5.0) days. Thirty-six (35.6\%) calves were fed waste milk, while the remaining

119 calves were fed either colostrum or whole milk.

120

C. difficile was detected by qPCR in 28 calves (30.4\%, 95\% Cl 21.2-40.9\%) (Fig. 1). Of the 28

121 samples that were positive for $C$. difficile on qPCR, 1 (3.6\%) was positive for Toxin B only, 14 (50\%) were

122 positive for binary toxin only, and 13 (46.4\%) were positive for both Toxin B and the binary toxin. None of

123 the organisms were identified as the NAP1/B1/027 ribotype. On 14 farms, there were both C. difficile-

124 positive and $C$. difficile-negative calves, whereas on the remaining farms, all of the calves were $C$. difficile-

125 negative. There were no farms where all samples were qPCR-negative but the pooled sample was culture-

126 positive. Neither calf age nor feeding of waste milk were significantly associated with the likelihood of

127 detecting $C$. difficile among the calves ( $O R=1.01, p=0.805$ and $O R=0.71, p=0.493$, respectively) (Fig. 1).

129 Effect of $C$. difficile status on microbiota diversity

130 Microbiota community structure of 87 calf fecal samples was assessed by sequencing and analyzing the

131 V4 region of the $16 \mathrm{~S}$ rRNA gene. Three samples were dropped from subsequent analyses because of low

132 coverage and four additional samples were dropped because there was not enough sample for qPCR 
analysis. Among the 80 remaining samples, 24 were positive for $C$. difficile by qPCR and 56 were negative

134 (Fig 1).

The relationship between $C$. difficile infection and microbial diversity of the gut microbiota was assessed. Since calves ranged in age, diet, and farm location, a linear mixed effects model was performed

137 to assess the relationship between $C$. difficile infection and alpha diversity by setting age as a fixed

138 variable and farm and feeding type as random-effect variables. The association between $C$. difficile status

139 and Shannon alpha diversity was not significant $(P=0.50)$ as determined by ANOVA when controlling for

140 age, diet, and farm location (Fig. 2). PERMANOVA was then used to test associations between C. difficile

141 infection status and beta diversity of the gut microbiome. Farm location alone explained most of the

142 variation in gut microbiota composition across samples using both Bray-Curtis $(P=1 \mathrm{e}-4 ; \mathrm{R} 2=0.43)$ and

143 weighted UniFrac ( $P=1 \mathrm{e}-4 ; \mathrm{R} 2=0.46)$ beta diversity metrics (Fig. 3, Fig. 4). Age and diet were not

144 significantly associated with gut microbiota composition after controlling for farm $(P>0.1)$. After

145 controlling for farm, age, and diet, C. difficile status was significantly associated with Bray-Curtis beta

146 diversity $(P=0.0077 ; \mathrm{R} 2=0.023)$, explaining $2.3 \%$ of the variation in gut microbiota composition. $C$. difficile

147 status was not significantly associated with weighted UniFrac beta diversity $(P=0.1934 ; \mathrm{R} 2=0.013)$ after

148 controlling for farm, age, and diet (Fig. 3). Some clustering by farm and by $C$. difficile status within farms

149 was apparent on principal coordinate analysis (Fig. 4).

151 Bacterial community composition

153 determined by beta diversity, we next sought to determine the specific bacterial taxa associated with $C$.

154 difficile infection. At the phylum level, there were no significant differences between bacterial

155 communities in C. difficile-positive and -negative samples (Fig. 5). The Firmicutes phylum predominated 
156 (57.1\% in C. difficile-positive samples and $51.4 \%$ in C. difficile-negative samples), followed by

157 Proteobacteria (17.1\% and 24.3\%), Bacteroides (16.7\% and 11.5\%), and Actinobacteria (8.1\% and 9.7\%).

159 samples by ANCOM occurred for Clostridioides. When considering LEFse analysis, there were four taxa

160 among the 19 taxa with average relative abundance greater than $1 \%$ that were statistically significantly

161 (Adj. P<0.1) associated with C. difficile status. Ruminococcus (gnavus group) (Adj. P=0.052),

162 Lachnoclostridium (Adj. P=0.060), Butyricicoccus (Adj. P=0.060), and Clostridium (sensu stricto 2) (Adj.

$163 P=0.064)$ were all found in higher abundance among $C$. difficile-positive calves than in $C$. difficile-negative

164 calves (Fig. 6). While not statistically significantly different among the two groups, levels of Lactobacillus,

165 Megasphaera, and Streptococcus were increased in C. difficile-positive samples, while levels of Blautia,

166 Fusobacterium, Tyzzerella, Enterobacteriaceae, Fecalibacterium, Dorea, and Collinsella were decreased.

168 interactions between bacteria using a co-occurrence analysis based on the Dice index. When considering

169 all levels of abundance, more co-occurrence of bacterial taxa appeared in the C. difficile-negative

170 samples, with $1,488(65.5 \%)$ highly (correlation coefficient $>0.6)$ and significantly $(p<0.01)$ correlated

171 genera pairs. Most co-occurrences were among members of the Firmicutes phylum (1295, 55.0\%).

172 However, members of Firmicutes also showed high co-occurrence with Actinobacteria and Bacteroidetes.

173 In the C. difficile-positive samples, there were fewer highly co-occurring genera, with 830 (73.3\%) highly

174 and significantly correlated genera pairs. When only considering taxa with levels of abundance greater

175 than 1\%, there were no significant differences in co-occurrence patterns (Fig. 7).

176

177 Discussion: 
178 In this study, we characterized microbial features associated with asymptomatic $C$. difficile colonization in

179 dairy calves. While the role of $C$. difficile in calf diarrhea remains equivocal (12), exploring the association

180 between this pathogen and the gut microbiome is important for understanding factors that affect gut

181 health and enteric diseases. While a number of studies have examined the epidemiology of $C$. difficile in

182 animals of veterinary importance, the association between the microbiome and C. difficile is only

183 beginning to be explored in dogs (9), horses (29), and pigs (30). Notably, in pigs, the presence of $C$.

184 difficile is associated with significantly reduced microbial diversity and increased levels of

185 enteropathogens associated with neonatal diarrhea (30).

187 variation in gut microbiota composition. However, even among calves from the same farm, there was

188 variability in both $C$. difficile colonization status and gut microbial diversity, suggesting, as have other

189 studies $(32,34)$, that the farm environment is only one of many competing influencers of the developing

190 calf gut microbiome. Neither diet nor age were significantly associated with microbiome composition

191 when controlling for farm, but this is almost certainly due to the small sample size within each farm and

192 the lack of within-farm variability in factors such as diet. When controlling for age, diet, and farm, we

193 noted a significant difference in beta diversity between $C$. difficile-positive and C. difficile-negative fecal

194 samples when considering the Bray-Curtis metric but not the unweighted UniFrac metric. While both of

195 these metrics are weighted by abundance, the latter metric weighs diversity by phylogenetic relationship.

196 Thus the lack of a significant difference when considering the weighted UniFrac metric suggests that,

197 while there may be a significant difference in the composition of microbial communities, the

198 differentially-abundant microbes might be closely related to one another. Indeed, all four genera

199 identified as differentially-abundant by LEfSe are members of the Clostridia class, with two belonging to

200 the Clostrideaceae family. 
While the lack of a consistent difference in alpha and beta diversity between $C$. difficile-positive

203 and $C$. difficile-negative samples suggests that the effect of $C$. difficile colonization on the gut microbiome

204 of calves is minimal, other findings suggest that $C$. difficile colonization is associated with a more

205 disrupted - but not dysbiotic - gut microbiome. C. difficile colonization was preferentially associated with

206 certain bacterial taxa of the class Clostridia that do have associations with dysbiosis. Notably, the

207 overrepresentation of Ruminococcus gnavus and Lachnoclostridia in C. difficile-positive calves point to the

208 possibility of an underlying imbalance in the gut microbiome. R. gnavus, a Gram-positive anaerobe that is

209 typically found in the gut of over $90 \%$ of healthy people at abundances less than $0.1 \%$, has been robustly

210 associated with inflammatory dysbiotic conditions such as Crohn's disease (35-37), allergic airway disease

211 (38), eczema (39), and spondyloarthritis (40). Dramatic blooms of R. gnavus occur in patients

212 experiencing flares of inflammatory bowel disease, with abundance levels that can peak at $69 \%$ of the gut

213 microbiota (37). Notably, this association appears to occur across species, as the gut microbiomes of both

214 infants (7) and piglets (30) colonized with C. difficile also had increased levels of Ruminococcus species,

215 including R. gnavus. Additionally, Ruminococcus was one of six bacterial genera in the gut microbiome

216 that predicted the occurrence of diarrhea in calves in another study (41). The increased relative

217 abundance of Clostridium sensu stricto and Lachnoclostridia in C. difficile-positive calves also points to the

218 possibility of a less healthy gut environment. An increased relative abundance of Clostridium sensu stricto,

219 which was also found in C. difficile-positive piglets (30), was associated with food allergies in infants (42)

220 and diarrhea in piglets (43). A tentative association between increased levels of Lachnoclostridia and

221 neoplasia of the gastrointestinal tract has been identified in people $(44,45)$. While no such association

222 has been explored in animals, the overrepresentation of this taxon in $C$. difficile-positive calves may be

223 the result of a more disrupted gut microbiota. However, it is also important to note that the increased

224 relative abundance of these taxa were only detected using LEfSe analysis and not ANCOM, which suggests

225 that the association is likely relatively weak. 
227 statistically significantly lower) levels in C. difficile-positive calves. Notably, Fecalibacterium, Dorea,

228 Enterobacteriaceae and Collinsella are among the most abundant genera in healthy pre-weaned calves

229 (46-49), and some of these taxa provide colonization resistance against $C$. difficile $(8,50)$. Their decreased

230 relative abundance in $C$. difficile-positive calves is thus also reflective of a more disrupted gut

231 microbiome. The decreased co-occurrence of bacterial taxa in C. difficile-positive calves compared to $C$.

232 difficile-negative calves when considering all levels of abundance may also corroborate the notion of a

233 slightly more disrupted gut microbiome in colonized calves. However, because the difference occurred

234 only in rare taxa (abundance $<1 \%$ ), this difference appears unlikely to result in dysbiosis.

238 with C. difficile (51) or diagnosed with inflammatory bowel disease $(52,53)$, and at higher levels in healthy 239 dairy calves compared to calves with diarrhea $(48,54)$. It is unclear why they were found at higher levels

240 in C. difficile-positive calves compared to C. difficile-negative calves. Butyricicoccus bacteria produce

241 butyrate, an important nutrient source for gut colonocytes and a beneficial driver of the immunological

242 maturation of the gut mucosa (55), and account for one of the most abundant genera in dairy calves 7

243 days after birth (56). The differential levels in calves compared to people with enteric disease may be due

244 to species-specific patterns of development of the neonatal gut. Species-specific differences may also

245 explain why C. difficile colonized calves had higher levels of Clostridial genuses but colonized puppies had

246 lower levels (9). While rumen development is minimal in pre-weaned calves, they are nevertheless

247 ruminants and thus have fundamentally different enteric physiologies and microbial ecologies compared

248 to true monogastric species. 
250 sampled calves may have obscured features of the microbiome that would otherwise have been

251 associated with $C$. difficile colonization. The cross-sectional nature of the study also precludes the

252 possibility of drawing any conclusions about the duration of colonization and its effect on an already

253 rapidly evolving gut microbiome. Finally, because we used qPCR to detect $C$. difficile in the calves' feces,

254 we were unable to detect non-toxigenic $C$. difficile. It is likely that toxigenic and non-toxigenic $C$. difficile

255 occupy a similar ecological niche and compete for similar resources within the gut microbiota; thus the

256 presence of non-toxigenic $C$. difficile could account for the lack of a significant difference in alpha

257 diversity and microbial composition between C. difficile-positive and C. difficile-negative calves. However,

258 we believe this possibility to be unlikely, as there were no samples that were negative on qPCR but came

259 from a farm where the pooled sample was positive for $C$. difficile on anaerobic culture.

\section{Conclusion}

262 The greatest source of variability in the calf microbiome was the farm, and there were few or no

263 statistically significant differences in alpha or beta diversity between $C$. difficile-positive and C. difficile-

264 negative calves. C. difficile colonization thus does not appear to be associated with dysbiosis or with

265 increased levels of enteropathogens that cause calf diarrhea. However, microbial community signatures -

266 including increased relative abundance of bacterial taxa that that have been associated with dysbiotic

267 states in other species and in people - suggest that the microbiota of $C$. difficile-colonized calves is more 268 disrupted than that of non-colonized calves. 
272 1. McGuirk SM. Disease management of dairy calves and heifers. Vet Clin North Am Food Anim

273 Pract. 2008 Mar;24(1):139-53. PubMed PMID: 18299036.

$2742 . \quad$ Foster DM, Smith GW. Pathophysiology of diarrhea in calves. Vet Clin North Am Food Anim 275 Pract. 2009 Mar;25(1):13-36, xi. PubMed PMID: 19174281.

3. Virtala AM, Mechor GD, Grohn YT, Erb HN. Morbidity from nonrespiratory diseases and mortality in dairy heifers during the first three months of life. Journal of the American Veterinary Medical Association. 1996 Jun 15;208(12):2043-6. PubMed PMID: 8707681.

279

280

281

282

4. Gulliksen SM, Jor E, Lie KI, Hamnes IS, Loken T, Akerstedt J, et al. Enteropathogens and risk factors for diarrhea in Norwegian dairy calves. J Dairy Sci. 2009 Oct;92(10):5057-66. PubMed PMID: 19762824.

5. Oikonomou G, Teixeira AG, Foditsch C, Bicalho ML, Machado VS, Bicalho RC. Fecal microbial diversity in pre-weaned dairy calves as described by pyrosequencing of metagenomic 16S rDNA. Associations of Faecalibacterium species with health and growth. PloS one. 2013;8(4):e63157. PubMed PMID: 23646192. Pubmed Central PMCID: 3639981.

6. Malmuthuge N, Chen Y, Liang G, Goonewardene LA, Guan le L. Heat-treated colostrum feeding promotes beneficial bacteria colonization in the small intestine of neonatal calves. J Dairy Sci. 2015 Nov;98(11):8044-53. PubMed PMID: 26342981.

7. Rousseau C, Levenez F, Fouqueray C, Dore J, Collignon A, Lepage P. Clostridium difficile colonization in early infancy is accompanied by changes in intestinal microbiota composition. J Clin Microbiol. 2011 Mar;49(3):858-65. PubMed PMID: 21177896. Pubmed Central PMCID: PMC3067754.

8. Zhang L, Dong D, Jiang C, Li Z, Wang X, Peng Y. Insight into alteration of gut microbiota in Clostridium difficile infection and asymptomatic C. difficile colonization. Anaerobe. 2015 Aug;34:1-7. PubMed PMID: 25817005.

9. Berry ASF, Kelly BJ, Barnhart D, Kelly DJ, Beiting DP, Baldassano RN, et al. Gut microbiota features associated with Clostridioides difficile colonization in puppies. PloS one. 2019;14(8):e0215497. PubMed PMID: 31469837. Pubmed Central PMCID: PMC6716646.

10. Houser BA, Soehnlen MK, Wolfgang DR, Lysczek HR, Burns CM, Jayarao BM. Prevalence of Clostridium difficile toxin genes in the feces of veal calves and incidence of ground veal contamination. Foodborne Pathog Dis. 2012 Jan;9(1):32-6. PubMed PMID: 21988399.

11. Knight DR, Thean S, Putsathit P, Fenwick S, Riley TV. Cross-sectional study reveals high prevalence of Clostridium difficile non-PCR ribotype 078 strains in Australian veal calves at slaughter. Appl Environ Microbiol. 2013 Apr;79(8):2630-5. PubMed PMID: 23396338. Pubmed Central PMCID: 3623178. 
12. Hammitt MC, Bueschel DM, Keel MK, Glock RD, Cuneo P, DeYoung DW, et al. A possible role for Clostridium difficile in the etiology of calf enteritis. Veterinary microbiology. 2008 Mar 18;127(3-4):34352. PubMed PMID: 17964088.

13. Malmuthuge N, Griebel PJ, Guan le L. The Gut Microbiome and Its Potential Role in the Development and Function of Newborn Calf Gastrointestinal Tract. Front Vet Sci. 2015;2:36. PubMed PMID: 26664965. Pubmed Central PMCID: PMC4672224.

14. Malmuthuge N, Guan LL. Understanding the gut microbiome of dairy calves: Opportunities to improve early-life gut health. J Dairy Sci. 2017 Jul;100(7):5996-6005. PubMed PMID: 28501408.

15. Kozich JJ, Westcott SL, Baxter NT, Highlander SK, Schloss PD. Development of a dual-index sequencing strategy and curation pipeline for analyzing amplicon sequence data on the MiSeq Illumina sequencing platform. Appl Environ Microbiol. 2013 Sep;79(17):5112-20. PubMed PMID: 23793624. Pubmed Central PMCID: PMC3753973.

16. Bolyen E, Rideout JR, Dillon MR, Bokulich NA, Abnet CC, Al-Ghalith GA, et al. Reproducible, interactive, scalable and extensible microbiome data science using QIIME 2. Nature Biotechnology. 2019 2019/08/01;37(8):852-7.

17. Callahan BJ, McMurdie PJ, Rosen MJ, Han AW, Johnson AJ, Holmes SP. DADA2: High-resolution sample inference from Illumina amplicon data. Nat Methods. 2016 Jul;13(7):581-3. PubMed PMID: 27214047. Pubmed Central PMCID: 4927377.

18. Katoh K, Standley DM. MAFFT multiple sequence alignment software version 7: improvements in performance and usability. Mol Biol Evol. 2013 Apr;30(4):772-80. PubMed PMID: 23329690. Pubmed Central PMCID: PMC3603318.

19. Price MN, Dehal PS, Arkin AP. FastTree 2--approximately maximum-likelihood trees for large alignments. PloS one. 2010 Mar 10;5(3):e9490. PubMed PMID: 20224823. Pubmed Central PMCID: 2835736.

20. Benjamini Y, Hochberg Y. Controlling the False Discovery Rate: A Practical and Powerful Approach to Multiple Testing. Journal of the Royal Statistical Society Series B (Methodological). 1995;57(1):289-300.

21. Bokulich NA, Kaehler BD, Rideout JR, Dillon M, Bolyen E, Knight R, et al. Optimizing taxonomic classification of marker-gene amplicon sequences with QIIME 2's q2-feature-classifier plugin. Microbiome. 2018 2018/05/17;6(1):90.

22. Quast C, Pruesse E, Yilmaz P, Gerken J, Schweer T, Yarza P, et al. The SILVA ribosomal RNA gene database project: improved data processing and web-based tools. Nucleic Acids Res. 2013;41(Database issue):D590-D6. PubMed PMID: 23193283. Epub 11/28. eng.

23. Bates D, Maechler M, Bolker B, Walker S. Fitting Linear Mixed-Effects Models Using Ime4. Journal of Statistical Software. 2015;67(1):1-48. 
24. Oksanen J, Blanchet F, Kindt R. Package, "vegan". 2015.

341

342

343

344

345

346

347

348

349

350

351

352

353

354

355

356

357

358

359

360

361

362

363

364

365

366

367

368

369

370

371

372

25. McMurdie PJ, Holmes S. phyloseq: an R package for reproducible interactive analysis and graphics of microbiome census data. PloS one. 2013;8(4):e61217. PubMed PMID: 23630581. Pubmed Central PMCID: PMC3632530.

26. Segata N, Izard J, Waldron L, Gevers D, Miropolsky L, Garrett WS, et al. Metagenomic biomarker discovery and explanation. Genome Biology. 2011 2011/06/24;12(6):R60.

27. Dice LR. Measures of the Amount of Ecologic Association Between Species. Ecology. 1945;26(3):297-302.

28. Wickham H. ggplot2: Elegant Graphics for Data Analysis. New York, NY: Springer-Verlag; 2016.

29. Schoster A, Kunz T, Lauper M, Graubner C, Schmitt S, Weese JS. Prevalence of Clostridium difficile and Clostridium perfringens in Swiss horses with and without gastrointestinal disease and microbiota composition in relation to Clostridium difficile shedding. Veterinary microbiology. 2019 Dec;239:108433. PubMed PMID: 31767096.

30. Grześkowiak $Ł$, Dadi TH, Zentek J, Vahjen W. Developing Gut Microbiota Exerts Colonisation Resistance to Clostridium (syn. Clostridioides) difficile in Piglets. Microorganisms. 2019 Jul 26;7(8). PubMed PMID: 31357520. Pubmed Central PMCID: PMC6723027. Epub 2019/07/31. eng.

31. O'Hara E, Kenny DA, McGovern E, Byrne CJ, McCabe MS, Guan LL, et al. Investigating temporal microbial dynamics in the rumen of beef calves raised on two farms during early life. FEMS Microbiology Ecology. 2020;96(2).

32. Haley BJ, Kim S-W, Salaheen S, Hovingh E, Van Kessel JAS. Differences in the Microbial Community and Resistome Structures of Feces from Preweaned Calves and Lactating Dairy Cows in Commercial Dairy Herds. Foodborne Pathogens and Disease. 2020 2020/08/01;17(8):494-503.

33. Gomez DE, Arroyo LG, Costa MC, Viel L, Weese JS. Characterization of the Fecal Bacterial Microbiota of Healthy and Diarrheic Dairy Calves. J Vet Intern Med. 2017;31(3):928-39. PubMed PMID: 28390070. Epub 04/07. eng.

34. Dill-McFarland KA, Breaker JD, Suen G. Microbial succession in the gastrointestinal tract of dairy cows from 2 weeks to first lactation. Sci Rep. 2017;7:40864-. PubMed PMID: 28098248. eng.

35. Henke MT, Kenny DJ, Cassilly CD, Vlamakis H, Xavier RJ, Clardy J. Ruminococcus gnavus, a member of the human gut microbiome associated with Crohn's disease, produces an inflammatory polysaccharide. Proceedings of the National Academy of Sciences. 2019;116(26):12672.

36. Joossens M, Huys G, Cnockaert M, De Preter V, Verbeke K, Rutgeerts $P$, et al. Dysbiosis of the faecal microbiota in patients with Crohn's disease and their unaffected relatives. Gut. 2011 May;60(5):631-7. PubMed PMID: 21209126. Epub 2011/01/07. eng. 
37. Hall AB, Yassour M, Sauk J, Garner A, Jiang X, Arthur T, et al. A novel Ruminococcus gnavus clade enriched in inflammatory bowel disease patients. Genome Medicine. 2017 2017/11/28;9(1):103.

38. Chua H-H, Chou H-C, Tung Y-L, Chiang B-L, Liao C-C, Liu H-H, et al. Intestinal Dysbiosis Featuring Abundance of Ruminococcus gnavus Associates With Allergic Diseases in Infants. Gastroenterology. 2018 2018/01/01/;154(1):154-67.

39. Zheng $\mathrm{H}$, Liang $\mathrm{H}$, Wang $\mathrm{Y}$, Miao M, Shi T, Yang F, et al. Altered Gut Microbiota Composition Associated with Eczema in Infants. PloS one. 2016;11(11):e0166026. PubMed PMID: 27812181. Pubmed Central PMCID: PMC5094743. Epub 2016/11/05. eng.

40. Breban M, Tap J, Leboime A, Said-Nahal R, Langella P, Chiocchia G, et al. Faecal microbiota study reveals specific dysbiosis in spondyloarthritis. Annals of the rheumatic diseases. 2017 Sep;76(9):1614-22. PubMed PMID: 28606969. Epub 2017/06/14. eng.

41. Ma T, Villot C, Renaud D, Skidmore A, Chevaux E, Steele M, et al. Linking perturbations to temporal changes in diversity, stability, and compositions of neonatal calf gut microbiota: prediction of diarrhea. The ISME Journal. 2020 2020/09/01;14(9):2223-35.

42. Ling Z, Li Z, Liu X, Cheng Y, Luo Y, Tong X, et al. Altered Fecal Microbiota Composition Associated with Food Allergy in Infants. Applied and Environmental Microbiology. 2014;80(8):2546-54.

43. Zhu JJ, Gao MX, Song XJ, Zhao L, Li YW, Hao ZH. Changes in bacterial diversity and composition in the faeces and colon of weaned piglets after feeding fermented soybean meal. Journal of medical microbiology. 2018 Aug;67(8):1181-90. PubMed PMID: 29923819. Epub 2018/06/21. eng.

44. Youssef O, Lahti L, Kokkola A, Karla T, Tikkanen M, Ehsan H, et al. Stool Microbiota Composition Differs in Patients with Stomach, Colon, and Rectal Neoplasms. Digestive Diseases and Sciences. 2018 2018/11/01;63(11):2950-8.

45. Liang JQ, Li T, Nakatsu G, Chen Y-X, Yau TO, Chu E, et al. A novel faecal Lachnoclostridium marker for the non-invasive diagnosis of colorectal adenoma and cancer. Gut. 2020;69(7):1248.

46. Klein-Jöbstl D, Schornsteiner E, Mann E, Wagner M, Drillich M, Schmitz-Esser S. Pyrosequencing reveals diverse fecal microbiota in Simmental calves during early development. Front Microbiol. 2014 2014-November-17;5(622). English.

47. Malmuthuge N, Griebel PJ, Guan le L. Taxonomic identification of commensal bacteria associated with the mucosa and digesta throughout the gastrointestinal tracts of preweaned calves. Appl Environ Microbiol. 2014 Mar;80(6):2021-8. PubMed PMID: 24441166. Pubmed Central PMCID: 3957634.

48. Tomassini L. Rectal microbiota dynamics in pre-weaned dairy calves depending on colostrum intake, presence of diarrhea and antibiotic treatment: Washington State University; 2015. 
49. Hennessy ML, Indugu N, Vecchiarelli B, Bender J, Pappalardo C, Leibstein M, et al. Temporal changes in the fecal bacterial community in Holstein dairy calves from birth through the transition to a solid diet. PloS one. 2020;15(9):e0238882.

50. Han SH, Yi J, Kim JH, Lee S, Moon HW. Composition of gut microbiota in patients with toxigenic Clostridioides (Clostridium) difficile: Comparison between subgroups according to clinical criteria and toxin gene load. PloS one. 2019;14(2):e0212626. PubMed PMID: 30785932.

51. Pérez-Cobas AE, Artacho A, Ott SJ, Moya A, Gosalbes MJ, Latorre A. Structural and functional changes in the gut microbiota associated to Clostridium difficile infection. Front Microbiol. 2014;5:335-. PubMed PMID: 25309515. eng.

52. Eeckhaut V, Machiels K, Perrier C, Romero C, Maes S, Flahou B, et al. Butyricicoccus pullicaecorum in inflammatory bowel disease. Gut. 2013;62(12):1745-52.

53. Devriese S, Eeckhaut V, Geirnaert A, Van den Bossche L, Hindryckx P, Van de Wiele T, et al. Reduced Mucosa-associated Butyricicoccus Activity in Patients with Ulcerative Colitis Correlates with Aberrant Claudin-1 Expression. Journal of Crohn's and Colitis. 2016;11(2):229-36.

54. Hang BPT, Wredle E, Dicksved J. Analysis of the developing gut microbiota in young dairy calves-impact of colostrum microbiota and gut disturbances. Tropical Animal Health and Production. 2020 2020/12/28;53(1):50.

55. Furusawa Y, Obata Y, Fukuda S, Endo TA, Nakato G, Takahashi D, et al. Commensal microbederived butyrate induces the differentiation of colonic regulatory T cells. Nature. 2013 2013/12/01;504(7480):446-50.

56. Alipour MJ, Jalanka J, Pessa-Morikawa T, Kokkonen T, Satokari R, Hynönen U, et al. The composition of the perinatal intestinal microbiota in cattle. Sci Rep. 2018;8(1):10437-. PubMed PMID: 29993024. eng. 


\section{$432 \quad$ Figure legends}

434 Figure 1: Distribution of age and C. difficile colonization status in 92 pre-weaned Holstein dairy calves

436 Figure 2: Alpha diversity of the gut microbiome in 86 pre-weaned Holstein dairy calves by C. difficile 437 colonization status

439 Figure 3: Beta diversity of the gut microbiome in 86 pre-weaned Holstein dairy calves by $C$. difficile 440 colonization status. A. Bray-Curtis beta diversity. B. Weighted UniFrac.

442 Figure 4: Bray-Curtis principal coordinate analysis (PCoA) of fecal samples from 86 pre-weaned dairy 443 calves by $C$. difficile colonization status and by farm

444

452 Figure 7. Analysis of co-occurrence among microbial lineages scored using the Dice index by $C$. difficile-

453 colonization status (positive and negative). Dice indexes are shown as a heat map for all genera present at 454 a level of abundance greater than $1 \%$ and with statistically significant $(p<0.01)$ co-occurrence are shown 455 as a heatmap. The degree of co-occurrence is shown by the color code at the bottom. 


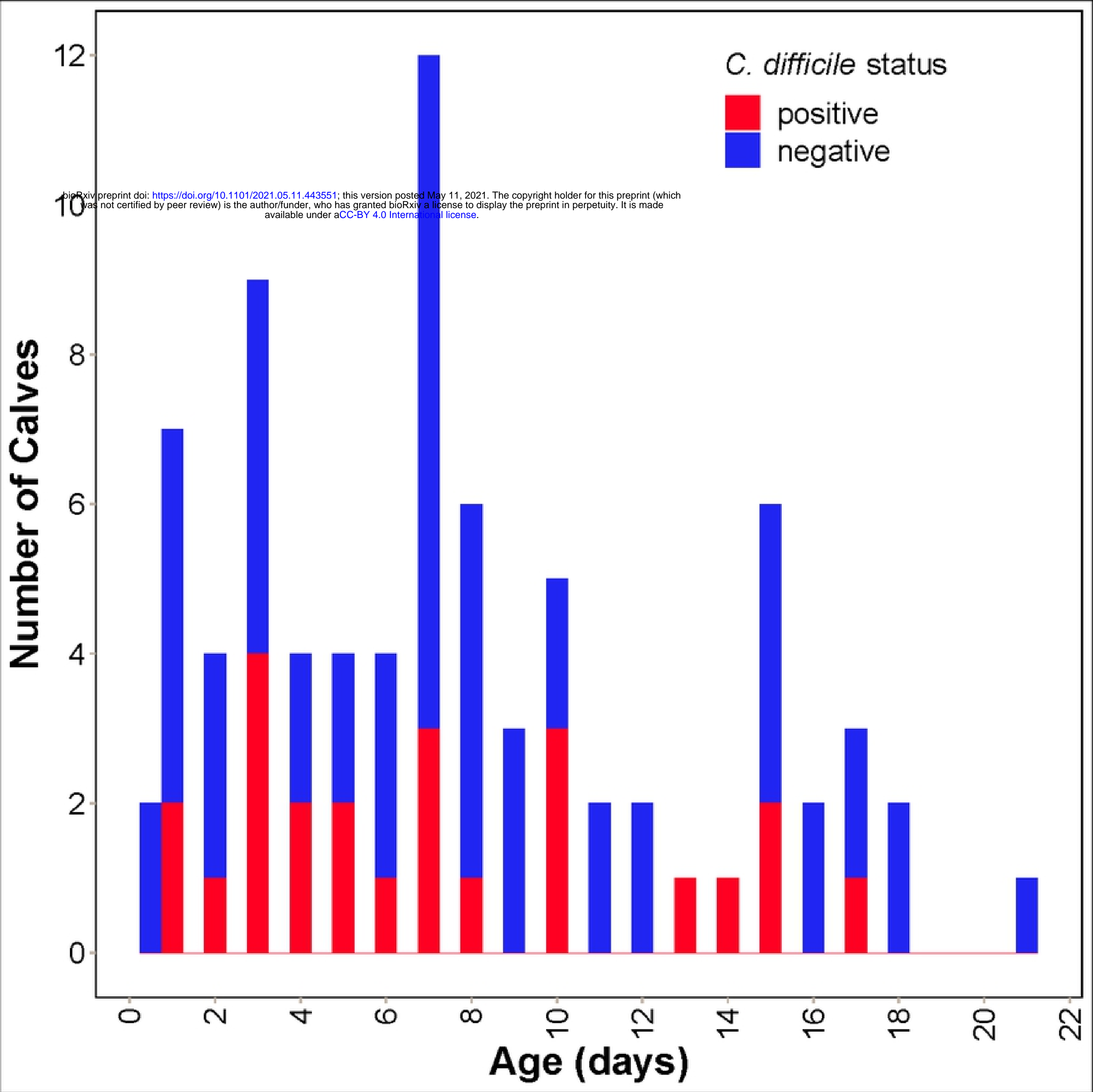

Figure 
5

waiv preprint doi: by peer review) is the author/funder, who has gr nted bo

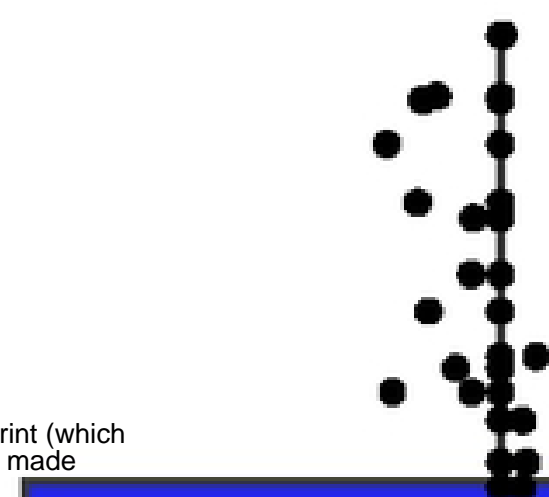

4

avaliable under aCC-B 8.0 International license.
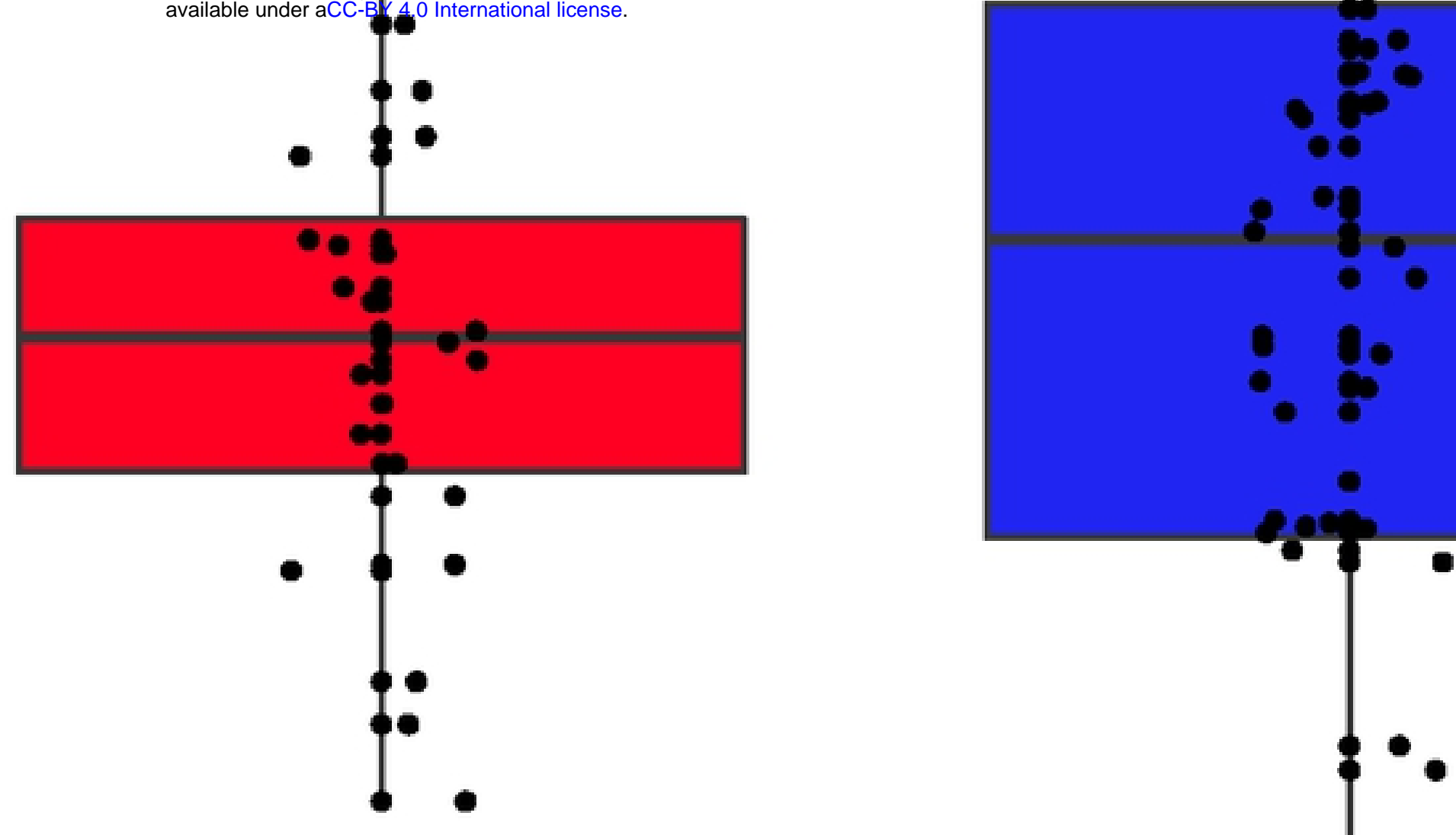

2

1
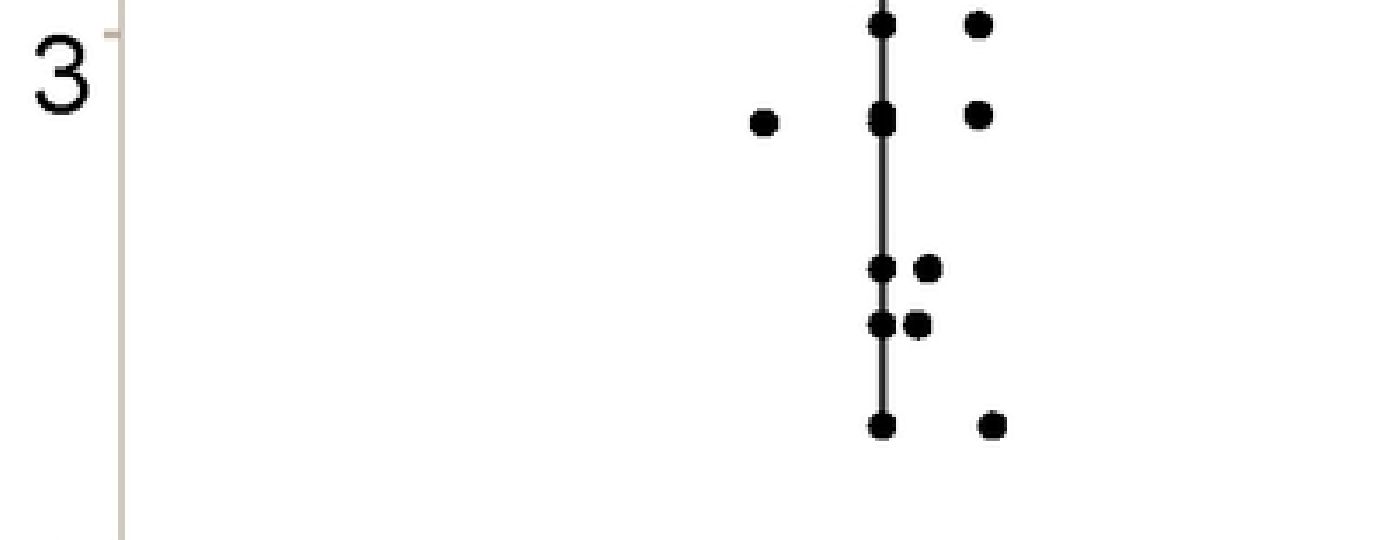

(1)

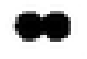

\section{Positive}

Negative

C. difficile status

Figure 
Bray-Curtis

1.00

0.50

bioRxiv preprint doi: https://doi.org/ $0.1101 / 2021.05 .11 .443551$; this version posted May 11, 2021. The copyright holder for this preprint (which

0.75

0.25
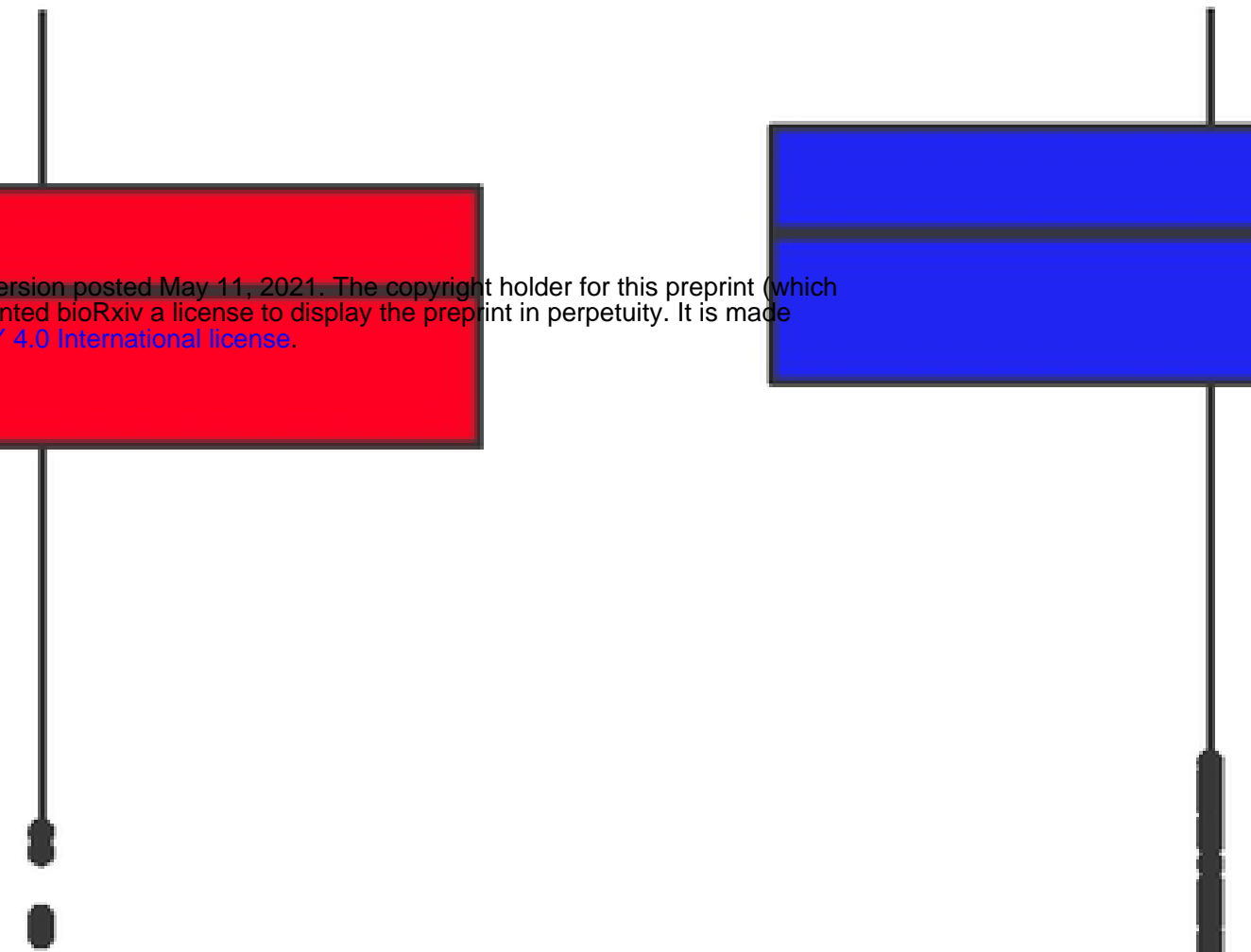

0.00
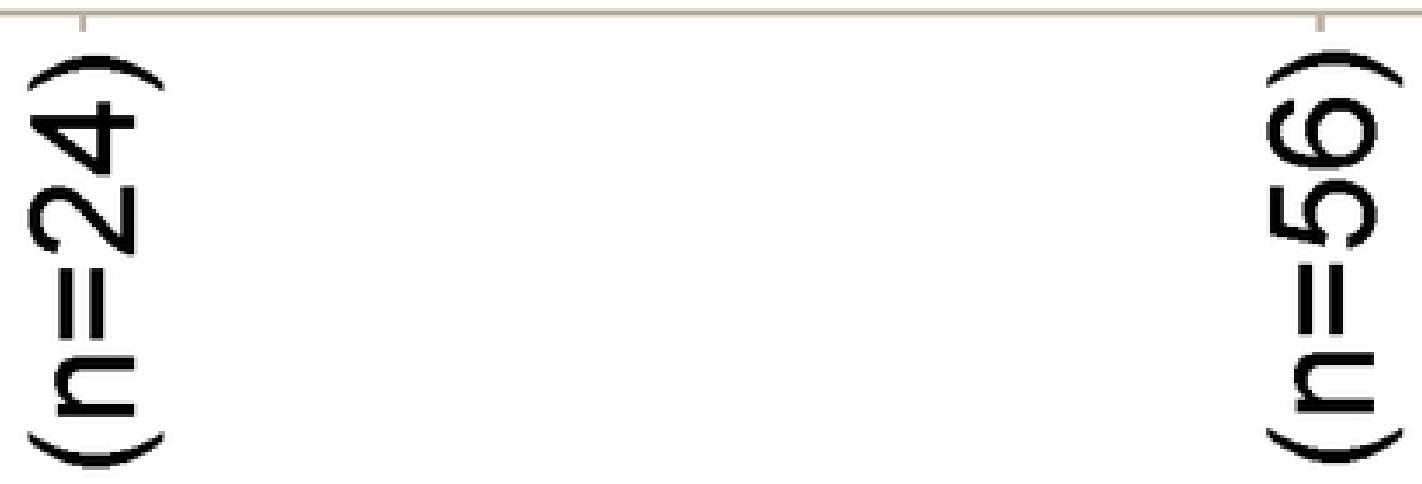

$\frac{1}{0}$

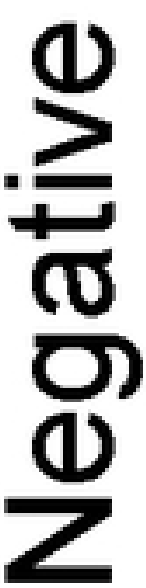

Figure 


\section{Weighted UniFrac}

1.00

bioRxiv preprint doi: https://doi.org/10.1101/2021.05.11.443551; this version posted May 11, 2021. The copyright holder for this preprint (which was not certified by peer review) is the author/funder, who has granted bioRxiv a license to display the preprint in perpetuity. It is made

0.75

0.50

0.25

0.00
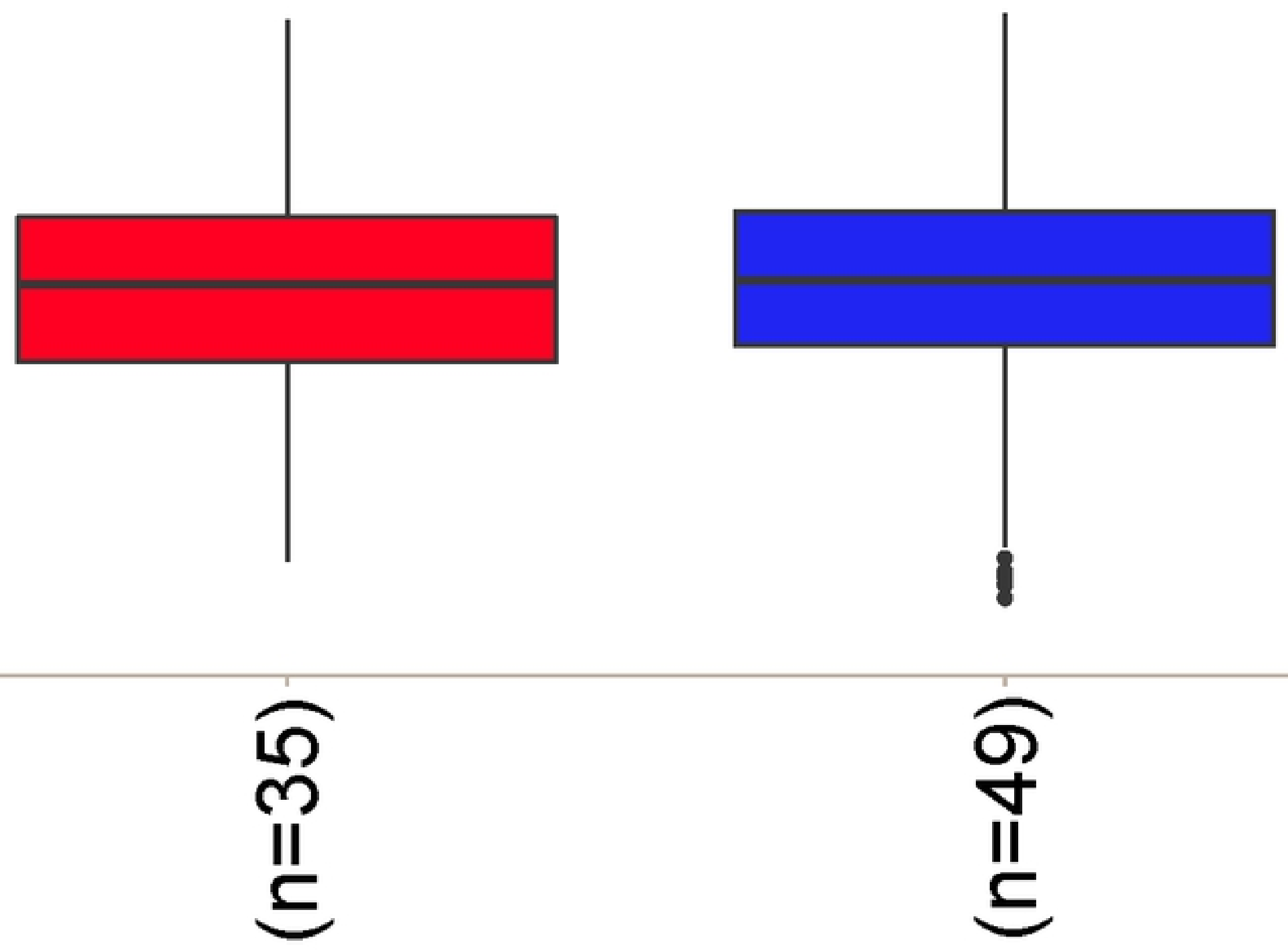

文

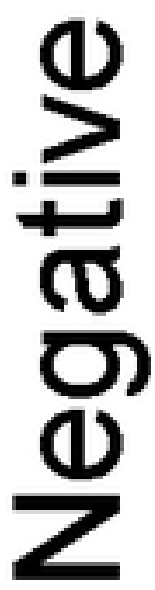

Figure 


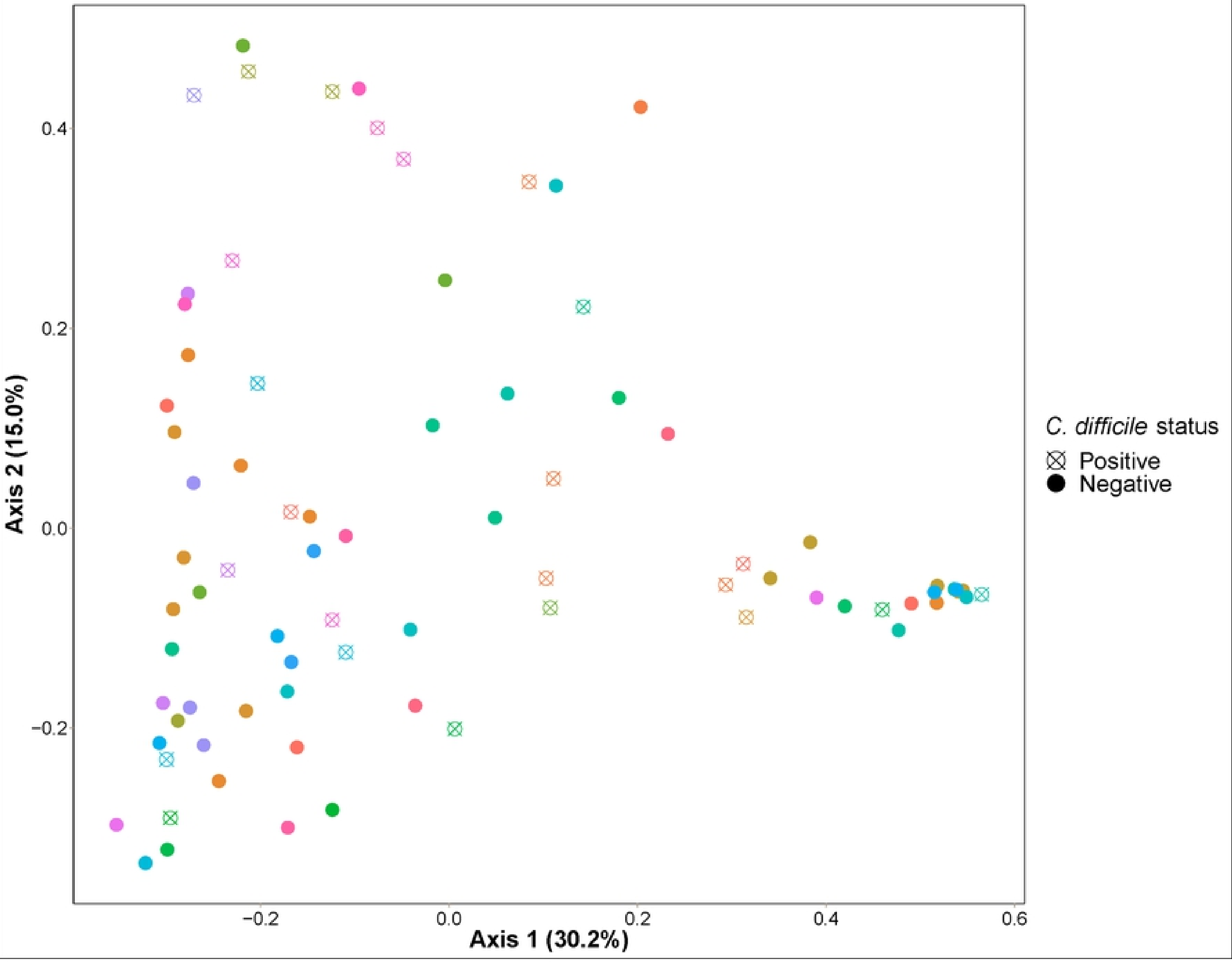

Figure 


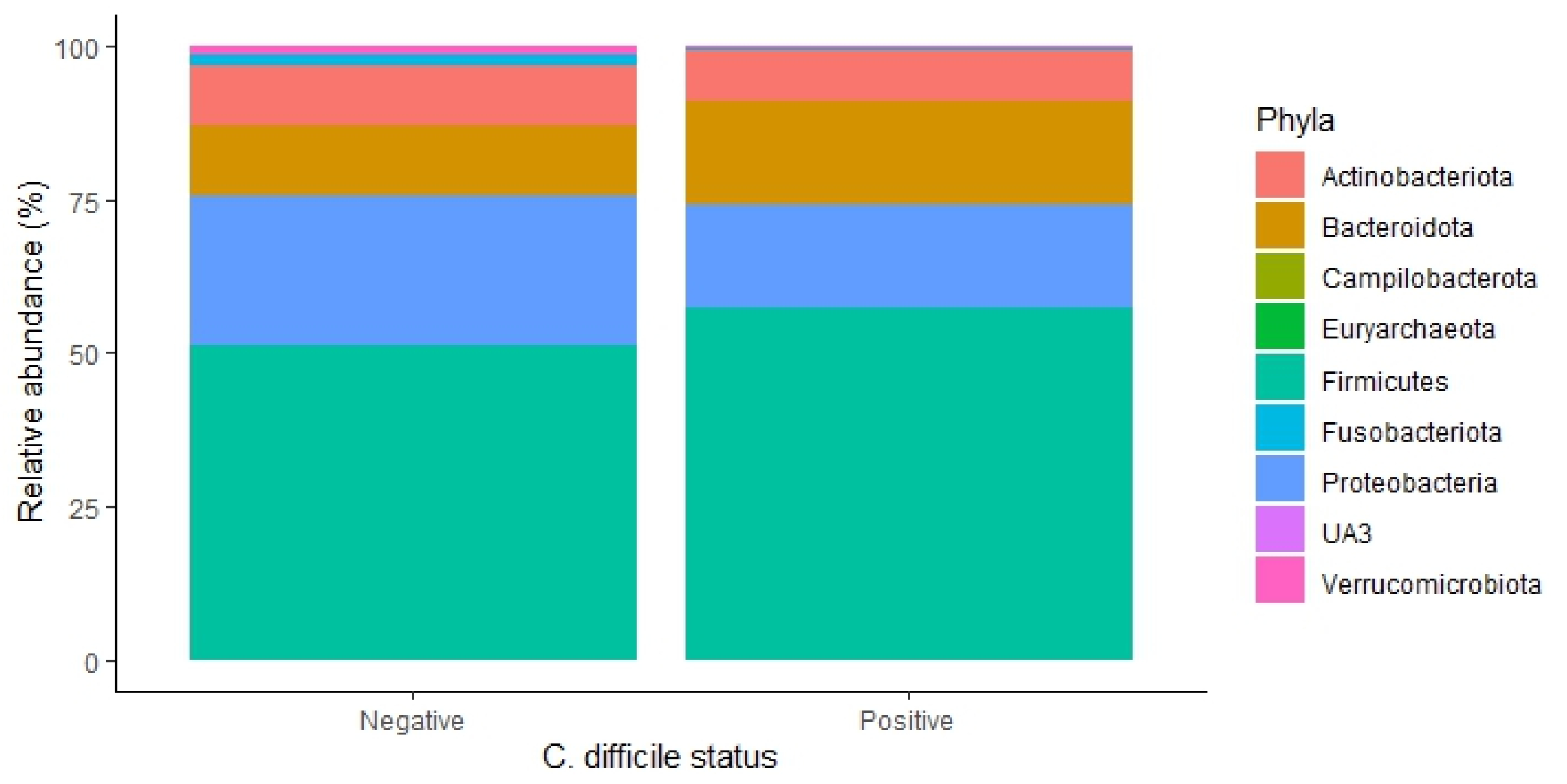

\section{Figure}



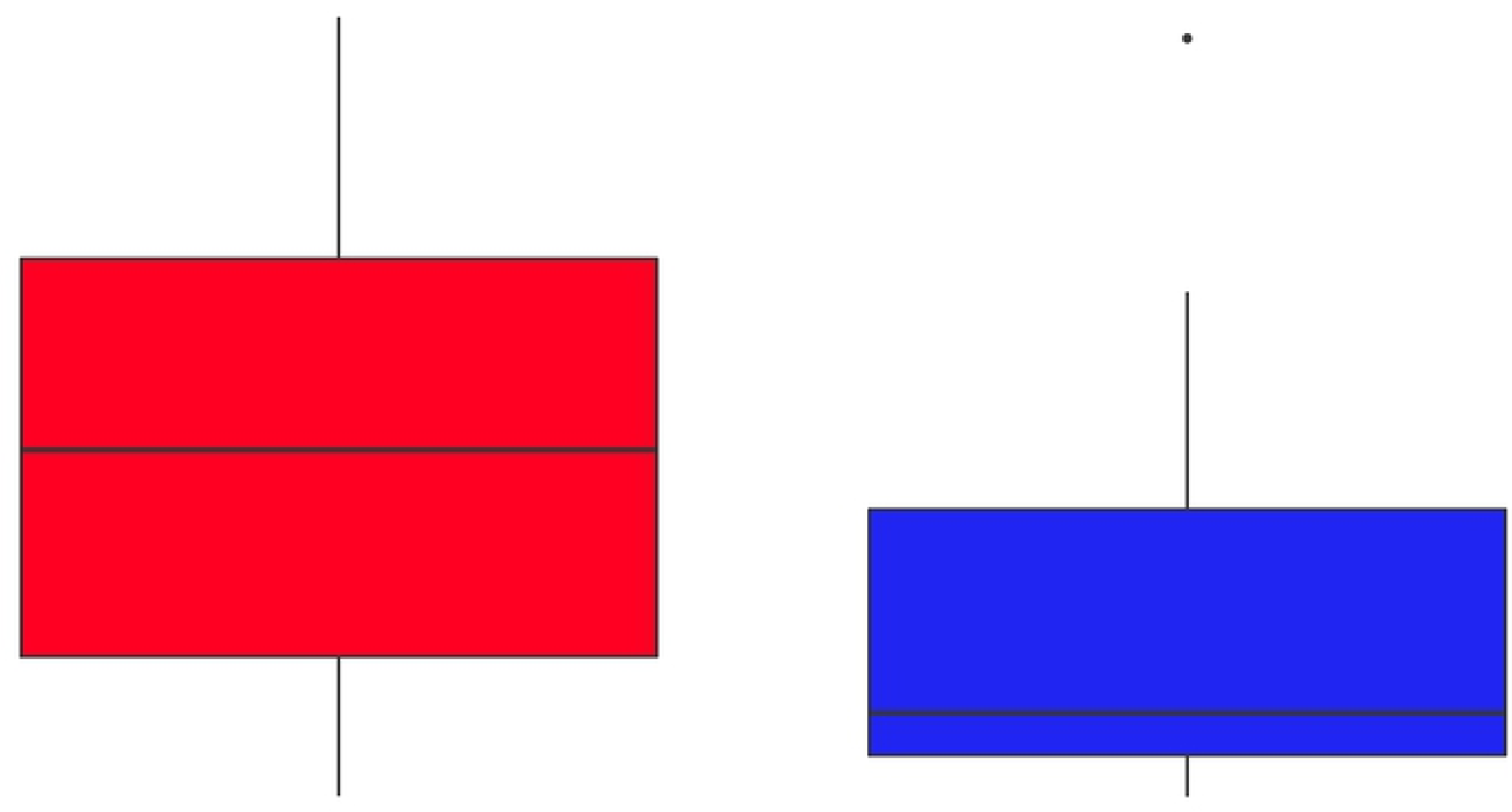

\section{Figure}




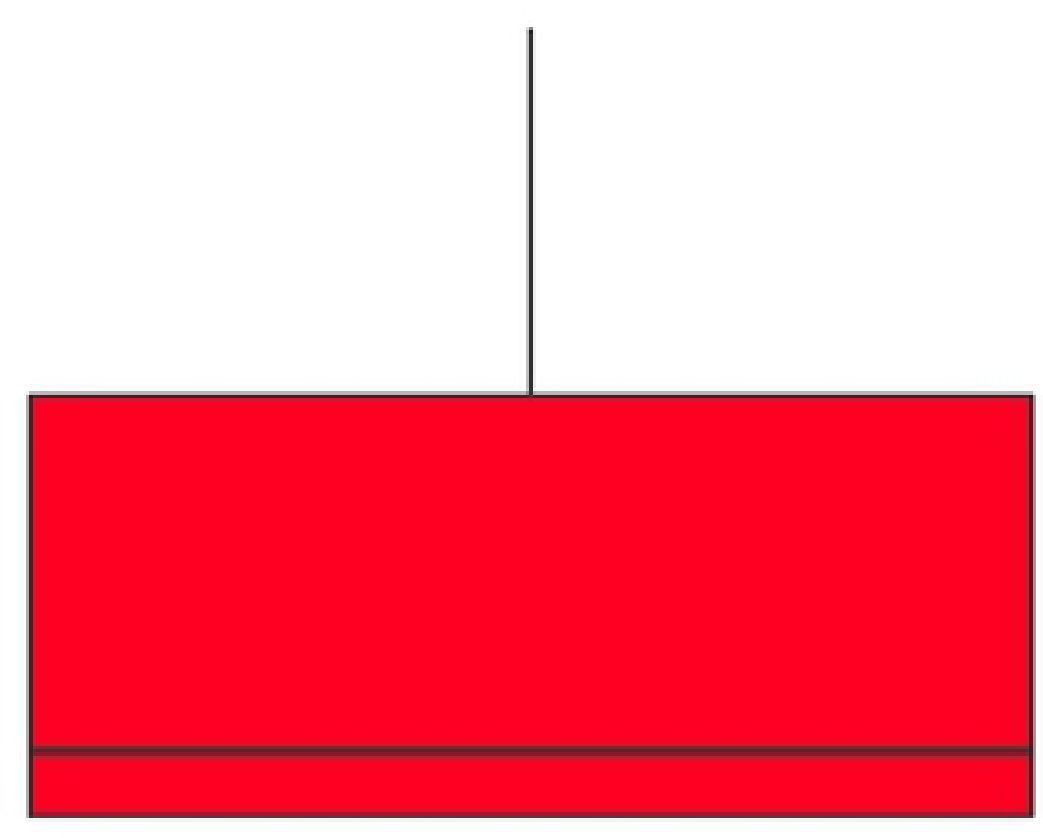

$\bullet$

:

\section{Figure}




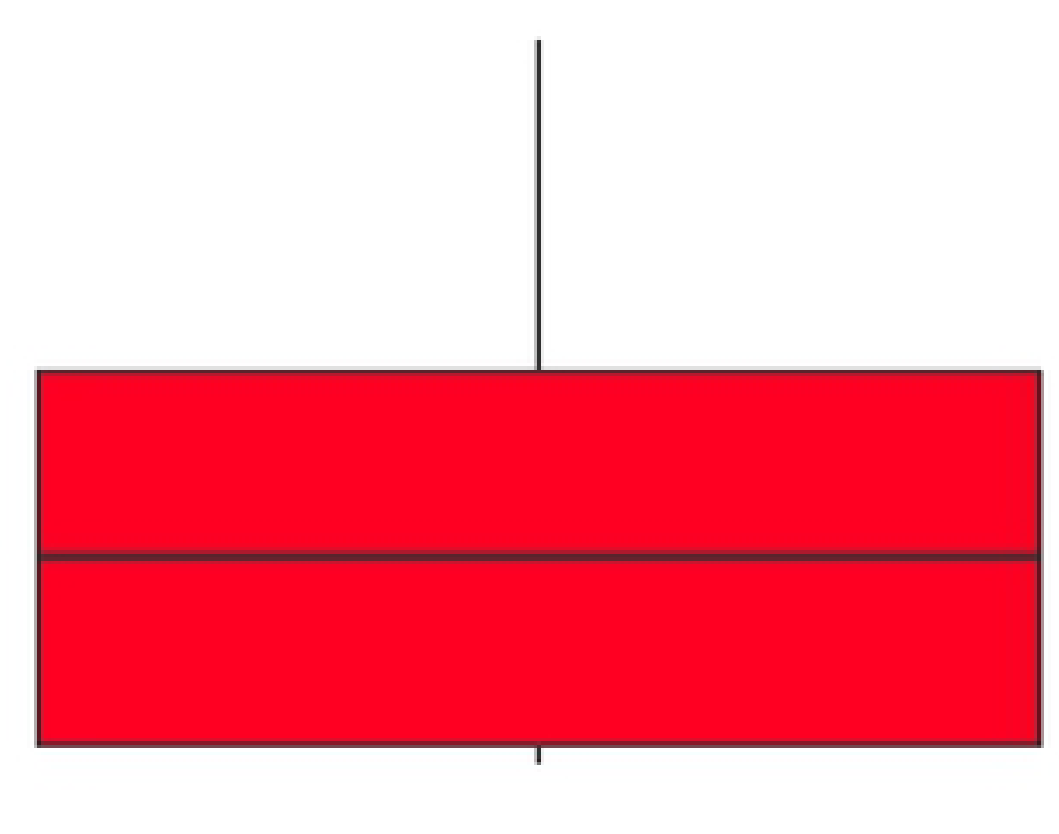




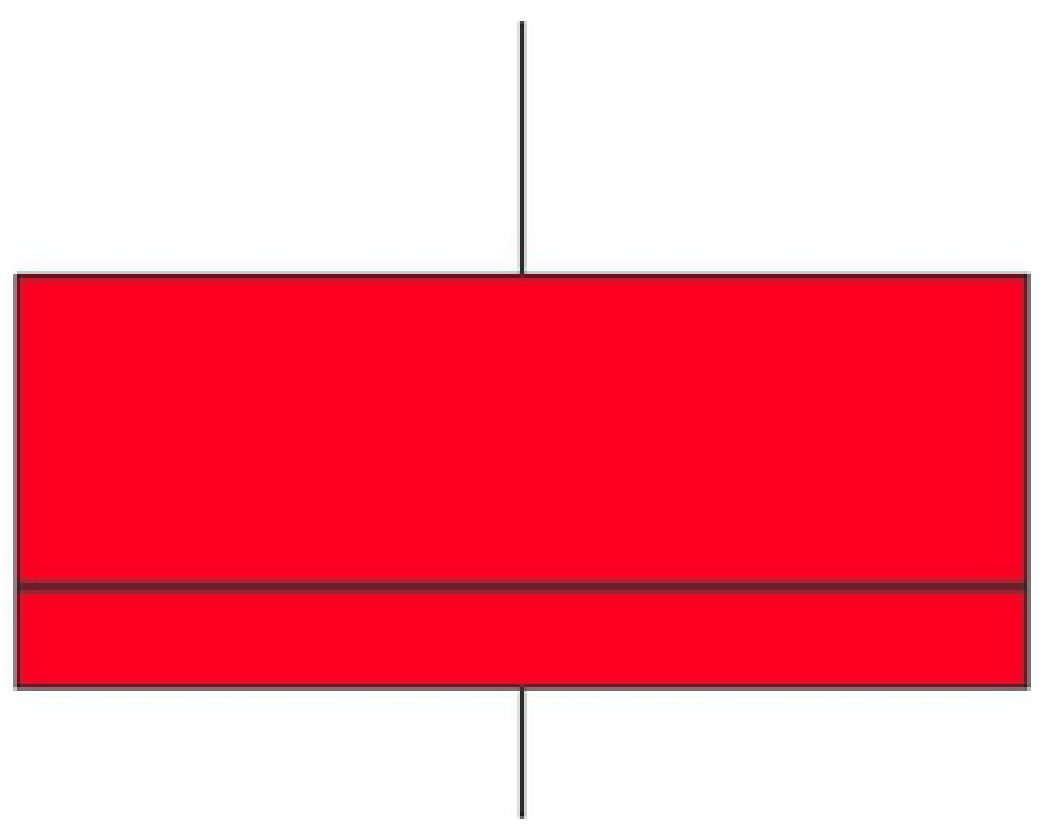




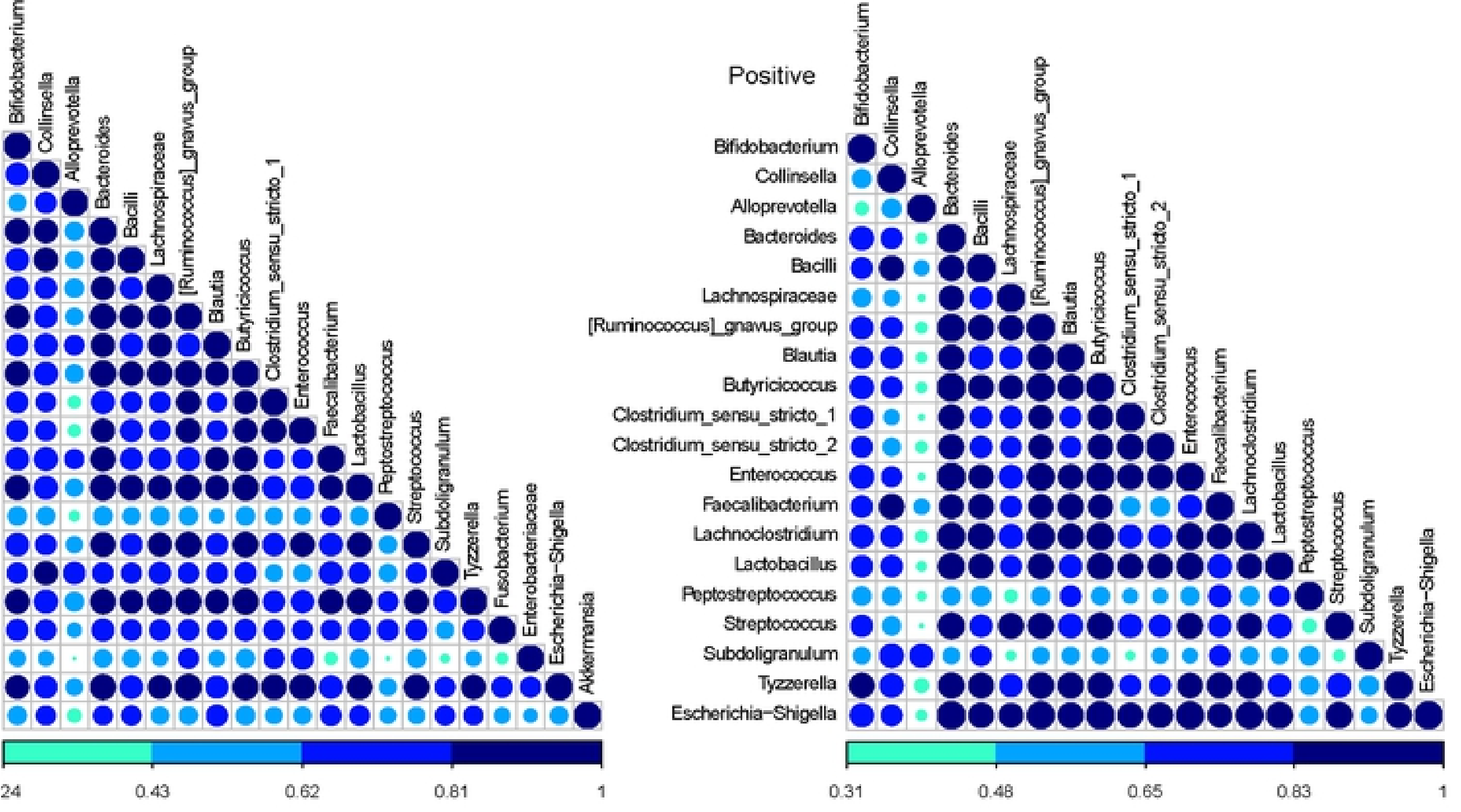

Figure 